\title{
Delivering perioperative care in integrated care systems
}

\author{
Authors: Anne-Marie Bougeard ${ }^{A}$, John Moore ${ }^{B}$
}

\section{In 2016, NHS England set up 10 integrated care systems} (ICSs) which aim to devolve some responsibility for delivery of health and social care services to local healthcare providers in partnership with local government, social care, primary care networks, and voluntary and charitable organisations. These are new ways of working and provide an opportunity to better integrate perioperative care across the entire pathway from the moment of contemplation of surgery through to recovery at home. This review describes the ways in which the aims of many ICS plans can be met with good perioperative care, and how clinicians can use this opportunity to make significant progress in improving outcomes for patients. We describe examples of initiatives in cancer pathways which are already proving successful and have caught the imagination of the local community at all levels, as well as examples of integrated perioperative care across the country which can be applied to other systems. We hope to demonstrate ways in which perioperative care can add value to a local health population given the right support and chance to deliver it.

KEYWORDS: Integrated care systems, perioperative care, prehabilitation, enhanced recovery, cancer

\section{Introduction}

In 2016, NHS organisations and councils in England joined to form 44 sustainability and transformation partnerships (STPS). ${ }^{1}$ Their aim was to plan care at a local level around the needs of their community and bring leaders from across the health and social care sector to deliver this. Over the following 2 years, a number of these STPs evolved into integrated care systems (ICSs) which foster closer collaboration with NHS organisations, councils, the voluntary sector and local community groups. ${ }^{2}$ ICS leaders have more freedom and devolved responsibility to manage their resources and performances to deliver health services to the specific needs of the population they serve. Latterly, there has been a natural evolution through the addition of further ICS areas, and by full devolution in some areas, including Greater Manchester and Surrey Heartlands.

Authors: ${ }^{A}$ anaesthetic registrar ST7 and perioperative medicine fellow, Derriford Hospital, Plymouth, UK; ${ }^{\text {B }}$ consultant in anaesthetics and intensive care medicine and clinical head of division for anaesthesia, Manchester University NHS Foundation Trust, Manchester, UK and clinical director for prehab, Greater Manchester Cancer, Manchester, UK
More than $30 \%$ of England's population is now covered by an ICS and the anticipation is that $100 \%$ coverage will follow by 2021 .

A recent survey of the membership of the Royal College of Anaesthetists ( $R C O A)$ revealed a degree of cynicism about ICSs and STPs, citing concerns about perceived lack of clinician engagement, and low levels of awareness of plans in their local area. The barriers to delivering perioperative care included difficulties in changing pathways, funding new initiatives, identifying patients earlier in the pathway to optimise and prehabilitate, and engaging other specialties in doing this. In 2018, the college commissioned an in-depth analysis of all published ICS plans to cross-reference them against the core principles of perioperative care. ${ }^{3}$ The report found common themes and demonstrated how perioperative care can address some of the aims set out in individual ICS plans, referencing example initiatives from other systems to demonstrate how this could be done.

The Centre for Perioperative Care (CPOC) aims to enhance collaborative, integrated, multidisciplinary working across the perioperative pathway, therefore it makes sense to work within ICSs to deliver these improvements. While the ICS plans are limited to England, the devolved nations have similar approaches in place to deliver improved population health across the health and social care sectors. ${ }^{4-6}$ This review will look at the opportunities offered by the new models of care for optimising integrated perioperative care pathways, using case examples to illustrate how this might be achieved.

\section{Relevance of surgery to local health systems}

High-quality perioperative care starts from the moment of contemplation of surgery and continues until the individual has made a full recovery. Surgery represents a significant burden on a health system: over one in 10 patients will undergo a surgical procedure per year. ${ }^{7}$ The RCoA launched their Perioperative Medicine Programme in 2015 highlighting the burden of highrisk surgery as a resource intense intervention, with $15 \%$ of patients accounting for $80 \%$ of deaths following surgery, and an even greater proportion suffering significant morbidity resulting in ongoing health service needs in the long term. ${ }^{8}$ Effective integration of care and consideration of the impact of surgery in the context of local population health is therefore very important.

\section{Structure of ICSs}

The ICS works on three levels.

> System: setting strategy, budget, workforce and degree of integration.

> Place: planning local services and delivery of secondary and community care. 
$>$ Neighbourhood and primary care networks: groups of general practitioner (GP) practices covering populations up to 50,000 providing a range of multidisciplinary services to the community, plus charitable and local government sector: $70-90 \%$ of ICS work will take place at this level. ${ }^{9}$

There are some key challenges with this model. Firstly, the ICS is not yet part of the statutory NHS arrangements and relies on good will between providers. Secondly, there will be changes to the current purchaser-provider relationship, which will impact the way in which services are commissioned. This is important in the context of a surgical pathway. Thirdly, regional level agreements are needed to cover networked services and ambulance trusts.

Let us consider perioperative care at primary care network level: many ICSs are setting up community hubs, which bring together services to support patients with long-term conditions. These hubs also provide support with lifestyle advice, and co-locate nursing, social workers, occupational therapists, social prescribers, community pharmacists among others. Grocott et al describe an ideal perioperative pathway, making use of the time between referral and surgery to optimise a patient's condition. ${ }^{10}$ Use of the community hub allows access these services early, and locally. Consequently, by the time decisions on surgery are made, some optimisation has already occurred. It would be hard to argue that this approach doesn't make sense, since at individual and population level these interventions are likely to be beneficial regardless of whether or not the patient ultimately undergoes surgery.

\section{Cancer pathways}

Recently, the RCoA, Macmillan and the National Institute for Health Research Cancer and Nutrition Collaboration launched guidance for prehabilitation for people with cancer. ${ }^{11}$ Most ICS plans include targets for cancer outcomes (early diagnosis, 1-year survival, quality of life after treatment for cancer and reducing unwarranted variation in outcomes), aims for improved population health through disease prevention and improving self-efficacy through social prescribing, public health campaigns and access to lifestyle interventions. Many ICSs also aim to streamline elective care pathways and reduce in-hospital treatment. The mainstay of treatment for solid tumours is surgery, therefore prehabilitation, cancer care and perioperative care are inextricably linked. The aims of prehabilitation (covered in detail elsewhere in this issue) align and overlap with those of ICSs and The NHS Long Term Plan. ${ }^{12}$ The ICS is a good way to deliver these objectives. An example is the PREPARE programme at Imperial College Healthcare NHS Trust which is offered to patients having major cancer surgery. ${ }^{13}$ This is a multimodal intervention offering an exercise programme, respiratory exercises, nutritional support, psychological support, smoking cessation, alcohol reduction support and enrolment into an enhanced recovery programme for surgery. The programme has reduced Clavien-Dindo grade II or more complications by $45 \%$ over 2 years and has won a number of awards, particularly for patient involvement.

\section{Keeping people out of hospital}

Many ICS priorities focus on care of its citizens out of hospital. While this is impractical in many surgical pathways, there are gains to be had in ambulatory surgery. The British Association of Day
Surgery directory lists index procedures and expected day case rates. ${ }^{14}$ The delivery of these targets is being evaluated by the Getting it Right First Time (GIRFT) programme for anaesthesia and perioperative medicine. It appears anecdotally that hospitals with high performing day surgery units have robust structures and processes in place across all perioperative pathways. ${ }^{15}$ Increasingly complex surgery is now being offered on a day case basis, including major joint replacements, nephrectomy, mastectomy and cholecystectomy (including emergency cases). Clearly this is attractive to an ICS, however, to maintain success and increase the proportion of surgery offered as a day case, there must be support in the community with outreach services, community services and multidisciplinary teams to manage patients in the community as they rehabilitate.

\section{Technology, digital records, communication}

Communication across a complex surgical pathway can be difficult. Our perioperative care leads encounter problems with lack of information coming from the community or from clinical records located at different hospitals. Many ICSs have recognised this gap as a barrier to continuity of care and have addressed this in their plans. Nottinghamshire have developed their capability for technology enabled care, including information sharing between staff and self-care support, as have Surrey Heartlands, and Dorset, who now have $95 \%$ of GPs using the same online system. Similar technology could be utilised in preassessment: patients can complete much of their own preassessment, which, with the referral, can allow triage to different pathways. The record can then accompany the patient through their inpatient stay, be contributed to by all professionals involved and continue with the transition out of hospital.

\section{Population health, self-care and the prevention agenda}

All ICS plans promote interventions to improve public health, including smoking cessation, alcohol reduction, improving physical activity and maintaining a healthy weight. Many ICS plans also emphasise the principle of self-management and self-efficacy when it comes to long-term health conditions. Personalised care, launched on the back of The NHS Long Term Plan earlier this year, looks at ways of better delivering on these principles. ${ }^{16}$ Contemplation of surgery represents a 'teachable moment' whereby an individual is more receptive to interventions to improve their health over the short and long term. Smoking cessation programmes at the time of surgery have been shown to have better long-term cessation rates than otherwise. ${ }^{17}$

Diabetes concerns all professionals along a perioperative pathway. Patients often arrive at preassessment with poor glycaemic control and require referral back to their GP for optimisation before elective surgery, or, if on an urgent pathway, have to proceed with suboptimal glycaemic control, risking complications. Many ICS plans prioritise diabetes care and management, for example, One Gloucestershire have a diabetes prevention programme, Buckinghamshire is integrating diabetes services across primary, acute and community care and Berkshire West is supporting patients with complex diabetes. Royal Bournemouth Hospital has a specialist preassessment clinic for patients with diabetes to plan glycaemic control before, during and after their surgery, backed up by e-learning and safe 
prescribing education for hospital clinicians. They have reduced average length of stay for inpatient surgery to parity with nondiabetic patients. Unpublished data from GIRFT demonstrate that there is wide variation in length of stay for diabetic patients across surgical specialties, therefore, there are significant gains to be had by integrating across the pathway. We have recent guidance from the National Confidential Enquiry into Patient Outcome and Death to support development of these initiatives. ${ }^{18}$

\section{But how do we do it?}

CPOC represents an opportunity to deliver consistent messaging about perioperative care from all constituent organisations. While the time is right to embed perioperative care into ICSs it is difficult to work out how to do it. Below are two case studies demonstrating how clinicians, researchers, patients and charities have come together to start this process. The commonality of these examples is with secondary care making the case for change and putting themselves forward to lead and make changes. They have had rich pickings and positive engagement, suggesting that there is will for this to happen. Experience from the vanguard sites reflects a willingness to take risks with different ways of working, with less focus on structure in favour of 'doing the right thing.' ${ }^{19}$ This is very much the remit of perioperative care.

\section{The Greater Manchester Cancer alliance}

Restructuring of the Greater Manchester (GM) healthcare system is supporting rapid transformation projects for patients undergoing major surgery such as Enhanced Recovery After Surgery Plus (ERAS+) and Prehab4Cancer.

\section{Organisation restructure}

Greater Manchester Combined Authority (GMCA) brings together the 10 boroughs of GM in a single organisation. In 2015, NHS organisations and councils signed the GM devolutional deal supporting GM taking charge of its own health and social care budget. In turn, the Greater Manchester Health and Social Care Partnership was developed to oversee the devolution of the health and social care services.

In 2016, both provider and commissioning boards for cancer services in GM agreed to set up a single system-wide cancer board, the Greater Manchester Cancer Board, which now oversees all cancer activity in the area, and reports into the Greater Manchester Health and Social Care Partnership. Through its cancer clinical pathways structure Greater Manchester Cancer can ensure that every patient in GM, no matter where they live, is afforded the same level of care following a diagnosis of cancer, while working to the NHS Cancer targets.

\section{ERAS+ and Prehab4Cancer}

The major surgical care for patients in GM, ERAS+, was developed at Manchester University NHS Foundation Trust (MFT) in a drive to reduce postoperative pulmonary complications (PPC), a common complication after major surgery affecting up to $25 \%$ of patients and associated with increased length of hospital stay and mortality. ${ }^{20}$

ERAS+ comprises prehospital prehabilitation (exercise, nutrition, smoking cessation, anaemia management and medical optimisation), a hospital-based postoperative respiratory care bundle (I COUGH), a prescription order set for oral healthcare, mobilisation and the use of incentive spirometry, which is built on ERAS stepped recovery model. ${ }^{21}$ The multidisciplinary patient education 'Surgery School' was developed to help support patient and family education about the ERAS+ programme and to help addresses the physical and psychological stressors associated with major surgery. The ERAS+ programme at Manchester Royal Infirmary was utilised with 1,000 patients using quality improvement methodology between 2014-16, reducing PPCs by $50 \%$ and hospital length of stay by 3 days, saving $£ 500,000$ each year. ${ }^{22}$

The scaling of the ERAS+ perioperative care across GM has been made significantly easier by the creation of the GMCA with senior oversight and GMCA sign-off supporting its uptake through the hospitals within GM. A Scaling Up Improvement grant from the Health Foundation was awarded to the ERAS+ programme in 2017 to support the implementation of ERAS+ across GM, and to understand its impact.

The GM ERAS+ programme is now a collaboration of multiprofessional clinical teams across the GM conurbation, aiming to improve outcomes for patients undergoing major surgery.

Most recently the preparation of patients in GM undergoing cancer surgery has been further enhanced by the Prehab4Cancer transformation project, supported by Greater Manchester Cancer which aims to facilitate prehab (exercise, nutritional and psychological support) for 2,000 patients over the next 2 years. ${ }^{23}$

\section{Wesfit $^{24}$}

In Wessex, the STP has part-funded a research study in collaboration with the University of Southampton NHS Foundation Trust and the Wessex Cancer Alliance. This will evaluate the impact of individualised exercise programmes and psychological support at community-based centres, on a number of outcomes:

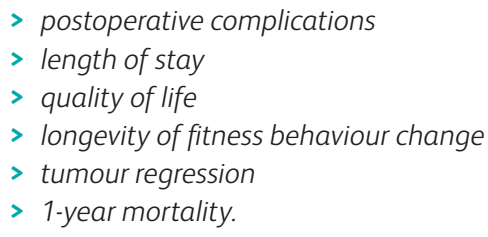

The project has been driven at community level with a range of partner organisations including private gyms, local charities and community centres, and has been co-designed with patients. The research will contribute to a body of evidence on the role of exercise prehabilitation interventions, in particular how they can be effectively delivered at community level. The WesFit model has had such appeal that other centres, including Plymouth, have now secured funding and engagement at local level to start recruiting patients.

\section{Conclusions}

There is a strong case that perioperative care adds value to a local health population given the right support and degree of integration. While in their relative infancy, it is clear that ICSs are 
here to stay, and although they will evolve, their establishment represents an opportunity to better deliver perioperative care across the whole of the pathway. The 'how-to' piece is the challenge. John F Kennedy said, 'Change is the law of life. And those who look only to the past or present are certain to miss the future. ${ }^{25}$ As a centre for perioperative care, we must equip our members with the confidence and consistent messaging that harnessing this opportunity is key. Perioperative care offers ways to deliver on the outcomes that politicians, clinicians and communities want. We should seize this opportunity.

\section{References}

1 NHS England. Sustainability and transformation partnerships. NHS www.england.nhs.uk/integratedcare/stps [Accessed 14 July 2019].

2 NHS England. Integrated care systems. NHS. www.england.nhs.uk/ integratedcare/integrated-care-systems [Accessed 14 July 2019].

3 Royal College of Anaesthetists. A teachable moment: delivering perioperative medicine in integrated care systems. London: RCoA, 2019. www.rcoa.ac.uk/document-store/delivering-pom-integratedcare-systems [Accessed 14 July 2019].

4 Realistic Medicine, 2019. www.realisticmedicine.scot [Accessed 14 July 2019].

5 Public Health Wales. Prudent healthcare. Public Health Wales, 2016. www.prudenthealthcare.org.uk/wp-content/uploads/2016/02/ Securing-Health-and-Wellbeing-for-Future-Generations1.pdf [Accessed 14 July 2019].

6 Department of Health Northern Ireland. Health and Wellbeing 2026 - delivering together. Department of Health Northern Ireland, 2017. www.health-ni.gov.uk/publications/health-andwellbeing-2026-delivering-together [Accessed 14 July 2019].

7 Abbott TEF, Fowler AJ, Dobbs TD et al. Frequency of surgical treatment and related hospital procedures in the UK: a national ecological study using hospital episode statistics. BJA 2017;119:249-57.

8 Royal College of Anaesthetists. Perioperative Medicine: The pathway to better surgical care. London: RCoA, 2015. www.rcoa. ac.uk/periopmed/vision-document [Accessed 14 July 2019].

9 British Medical Association. Integrated Care Systems (ICSs). BMA 2019. www.bma.org.uk/collective-voice/policy-and-research/nhsstructure-and-delivery/nhs-structures-and-integration/integratedcare-systems [Accessed 14 July 2019]

10 Grocott MPW, Plumb JOM, Edwards M, Fecher-Jones I, Levett DZH. Re-designing the pathway to surgery: better care and added value. Perioper Med (Lond) 2017;6:9.

11 Macmillan Cancer Support, NIHR Cancer and Nutrition Collaboration, Royal College of Anaesthetists. Prehabilitation for people with cancer: Principles and guidance for prehabilitation within the management and support of people with cancer. Macmillan, 2019. www.macmillan.org.uk/assets/prehabilitationguidance-for-people-with-cancer.pdf [Accessed 14 July 2019].

12 NHS. The NHS Long Term Plan. NHS, 2019. www.longtermplan.nhs. uk [Accessed 14 July 2019].

13 Imperial College Healthcare NHS Trust. The PREPARE programme. Imperial College Healthcare NHS Trust, 2019. www.imperial.nhs. uk/our-services/cancer-services/oesophago-gastric-cancer/prepareprogramme [Accessed 14 July 2019].

14 British Association of Day Surgery. BADS directory of procedures, 6th Edn. London: BADS, 2019.

15 Snowden C. Personal communication, July 2019.

16 NHS England. Personalised care. NHS, 2019. www.england.nhs.uk/ personalisedcare [Accessed 14 July 2019].

17 Lee SM, Landry J, Jones PM, Buhrmann O, Morley-Forster P. Long term quit rates after a preoperative smoking cessation randomized controlled trial. Anesth Analg 2015;120:582-7.

18 National Confidential Enquiry into Patient Outcome and Death. Perioperative diabetes: Highs and lows. London: NCEPOD, 2018. www.ncepod.org.uk/2018pd.html [Accessed 14 July 2019].

19 NHS Providers. The journey to integrated care systems. NHS. https://nhsproviders.org/provider-voices-integrated-care [Accessed 14 July 2019].

20 Mazo V, Sabate S, Canet ] et al. Prospective external validation of a predictive score for postoperative pulmonary complications. Anaesthesiology 2104;121:219-31.

21 Cassidy MR, Rosencrantz P, McCabe K, Rosen JE, McANeny D. I COUGH: reducing postoperative pulmonary complications with multidisciplinary patient care program. JAMA Surg 2013;148:740-5.

22 Moore JA, Conway DH, Thomas N, Cummings D, Atkinson D. Impact of a peri-operative quality improvement programme on postoperative pulmonary complications. Anaesthesia 2017;72:317-27.

23 Greater Manchester Cancer. Prehab4cancer. Greater Manchester Cancer. https://gmcancer.org.uk/our-areas-of-work/prehab4cancer [Accessed 14 July 2019].

24 ClinicalTrials.gov. The Wessex Fit-4-Cancer Surgery Trial (WesFit). ClinicalTrials.gov Identifier: NCT03509428.

25 Kennedy JF. Public papers of the presidents of the United States: John F. Kennedy: 1963: containing the public messages, speeches, and statements of the president, January 1 to November 22, 1963. Washington: United States Government Printing Office, 1963:517.

Address for correspondence: Dr Anne-Marie Bougeard, Derriford Hospital, Derriford Road, Plymouth, Devon PL6 8DH, UK. Email: anne-marie.bougeard@nhs.net 